\title{
Escala de Instabilidade Emocional para a Segurança Pública
}

\author{
Marcela dos Santos Reis - Universidade Salgado de Oliveira, Rio de Janeiro, Brasil \\ Cristiane Faiad - Universidade Salgado de Oliveira, Rio de Janeiro, Brasil
}

\begin{abstract}
Resumo
O presente trabalho expõe a construção e apresentação de evidências de validade de um instrumento de medida de instabilidade emocional para uso na área da segurança pública. Para tal, foram realizados dois estudos com amostras de profissionais de segurança pública do estado do Rio de Janeiro. O primeiro estudo apresentou evidências de validade baseadas no conteúdo. No segundo, a análise da estrutura interna apontou a existência de um fator e de quatro facetas denominados, respectivamente, instabilidade emocional, depressão, ansiedade, controle emocional e irritabilidade. A análise da consistência, por meio do alfa de Cronbach, evidenciou bons índices de precisão. A avaliação de convergência, realizada com o teste EFN, possibilitou observar correlações fortes entre os fatores gerais de ambas as escalas. Tais dados apontam evidências de validade para a nova medida e abrem caminho para novas pesquisas que tenham como foco a interface da psicologia com a segurança pública.

Palavras-chave: Instabilidade emocional; Avaliação psicológica; Cinco grandes fatores; Construção de teste
\end{abstract}

Scale to Measure Emotional Instability for Public Safety

\begin{abstract}
This work presents the construction and conveyance of validity evidence of an emotional instability measurement instrument to be used in the public safety sector. For this purpose, two studies were performed with subjected public safety professionals of the State of Rio de Janeiro. The first study presented valid evidence based on content. In the second study, the analysis of the internal structure appointed the existence of one factor and four facets, respectively named emotional instability, depression, anxiety, emotional control and irritability. The consistency analysis, using the Cronbach's alpha coefficient, has shown evidence of good accuracy rates. The convergence evaluation, performed with the EFN (Factorial Neuroticism Scale), has allowed observation of strong correlations between the general factors of both scales. Such information provides valid evidence for the new scale, and opens new research paths which focus on psychological interface with public safety.

Keywords: Emotional instability; Psychological evaluation; Big five factors; Test construction.
\end{abstract}

Escala de Inestabilidad Emocional para la Seguridad Pública

\begin{abstract}
Resumen
Esta investigación expone la construcción y la presentación de evidencias de validez de un instrumento de medida de inestabilidad emocional para utilización en el área de la seguridad pública. Para ello fueron realizados dos estudios con muestras de profesionales de seguridad pública de Río de Janeiro-Brasil. El primer estudio presentó evidencias de validez basadas en el contenido. En el segundo, el análisis de la estructura interna apuntó la existencia de un factor y de cuatro facetas denominadas respectivamente inestabilidad emocional, depresión, ansiedad, control emocional e irritabilidad. El análisis de la consistencia, por medio del alfa de Cronbach, evidenció buenos índices de precisión. La evaluación de convergencia, realizada con la prueba EFN, permitió observar fuertes correlaciones entre los factores generales de ambas escalas. Estos datos muestran evidencias de validez para la nueva medida y abren el camino hacia nuevas investigaciones que tengan como tema la interfaz de la psicología con la seguridad pública.

Palabras clave: Inestabilidad emocional; Evaluación psicológica; Cinco grandes factores; Construcción de test.
\end{abstract}

A segurança pública, conforme disciplina o artigo 144 da Constituição Brasileira, compreende, dentre outras atividades, a preservação da ordem pública, da integridade física dos cidadãos e do patrimônio (Brasil, 1988). O dever, apresentado aos agentes da área, implica a exposição direta a riscos pessoais e exige destes um conjunto de capacidades e habilidades a serem constantemente aplicadas em sua prática profissional. Embora necessários, constantemente se questiona quais são esses requisitos ou competências importantes para que profissionais da área da segurança pública desempenhem bem suas atividades.
Buscando responder, dentre outras questões, quais características devem ser consideradas como necessárias para a execução das atividades inerentes à área da segurança pública no Brasil, foram desenvolvidas análises profissiográficas (por exemplo, Pasquali, Cabral, Figueira, Rodrigues \& Moura, 2002; Thadeu \& cols., 2008). Tais estudos constataram ser o controle emocional um atributo indispensável ao bom desempenho de profissionais dessa área, concluindo pela necessidade de mensurar tal requisito quando do ingresso no serviço.

Outros estudos também indicam que altos escores em instabilidade emocional aumentam a dificuldade do 
exercício laboral em áreas críticas, como a segurança pública (Black, 2000; Ho, 2001). O uso de armamento letal como uma das atribuições legais de tais profissionais explica, em parte, a importância atribuída a esse quesito (Thadeu \& cols., 2008).

Neste contexto, é demandado que processos seletivos para ingresso na área da segurança pública apresentem cada vez mais melhorias no que tange à avaliação psicológica realizada, com a oferta de instrumentos psicológicos com qualidade psicométrica (Moura, 2008). Apesar dessa necessidade, observa-se que psicólogos da área têm enfrentado algumas dificuldades, dentre elas a escassez de instrumentos psicológicos que norteiem esses processos de avaliação (Noronha \& Alchieri, 2005).

Além da escassez de instrumentos e da necessidade do uso de medidas específicas, os psicólogos que trabalham com processos seletivos na área de segurança pública têm enfrentado outros desafios em sua prática profissional. Os obstáculos se referem a situações como, por exemplo: a) falta de instrumentos que contemplem em suas amostras de normatização participantes da área de segurança pública; b) poucos investimentos em pesquisas que contribuam para a compreensão da área (Moura, 2008) e c) a exposição de instrumentos psicológicos veiculados na internet, restringindo ainda mais as possibilidades de seu uso em processos públicos de seleção (Faiad, 2007; Thadeu, Kistenmacker \& Reis, 2009). Neste sentido, a escassez de medidas disponíveis para avaliar a estabilidade emocional de candidatos aos cargos da área de segurança pública aponta para a necessidade de construção de instrumentos específicos que contemplem as peculiaridades da função.

Estudos relacionados à seleção de pessoal para emprego na área da segurança pública evidenciam o imperativo da adequação psicológica para o bom desempenho na área e corroboram a necessidade da construção de medidas específicas para essa população. Dentre esses estudos, podemos citar o levantamento realizado por Cochrane, Tell e Vandecreek (2003). Esses autores constatam que, na realidade estadunidense, a maioria dos testes disponíveis é validada com amostras de origem clínica, o que os torna inadequados para o contexto de seleção de pessoal. Do mesmo modo, apontam para a falta de instrumentos que compreendam amostras de policiais em seus estudos, o que reduziria a validade dos testes utilizados em tal contexto. Nos resultados do trabalho em questão, os autores apontaram que traços de neuroticismo, extroversão e abertura, levantados por meio do NEO PI-R, apresentaram valor preditivo para o desempenho de policiais.

No Brasil, Thadeu (2011) pesquisou o quanto a personalidade e a cognição seriam bons preditores de desempenho de alunos de um curso da área de segurança pública. Foi observado que os escores nos testes psicológicos, realizados para admissão aos cursos de formação, contribuiram pouco para a explicação do desempenho dos alunos durante os mesmos. Segundo a autora, tal fato pode estar relacionado à validade dos instrumentos utilizados na seleção, considerando que muitas dessas medidas teriam seu conteúdo tornado público de forma indevida, permitindo que candidatos estivessem habituados aos padrões de respostas esperados. Uma das consequências possíveis desse fato seria a menor discriminação desses sujeitos.

Quanto à ênfase no construto de instabilidade emocional, essa é devida ao fato de que tal fator representa, junto à literatura da área (Bishop \& cols., 2001; Black, 2000) e nos estudos profissiográficos realizados (Pasquali \& cols., 2002; Thadeu \& cols., 2008), um conjunto de características julgadas de importância para o bom desempenho de profissionais de segurança pública. A magnitude da característica de instabilidade emocional também pode ser mensurada pela quantidade de estudos realizados em diferentes campos, tais como, organizacional, escolar e clínico (Hampson, 2012; Wiggins \& Pincus, 1992).

Nesse sentido, instabilidade emocional ou neuroticismo, no modelo dos cinco grandes fatores (CGF), contempla as facetas de ansiedade, raiva/hostilidade, depressão, embaraço/constrangimento, impulsividade e vulnerabilidade e pode ser definida como uma forte propensão para, de um modo geral, experienciar emoções negativas (McCrae \& Costa, 1989). Ela também caracteriza sujeitos que tendem a perceber o mundo como perigoso e ameaçador, sendo mais suscetíveis a manifestações de baixo autocontrole e irracionalidade (McCrae, 2006). Dessa forma, sujeitos caracterizados como emocionalmente instáveis, ou seja, aqueles que apresentam altos escores no fator, tendem à labilidade emotiva e mostram-se ansiosos, tensos e propensos a reações emocionais, além de apresentarem maior dificuldade na resolução de problemas (Sangil \& Weed, 2007).

Com o conjunto de informações apresentadas até o momento, foi possível compreender que a acentuação do traço de instabilidade emocional, acarreta prejuízos aos profissionais, à área da segurança pública e ao cidadão. Também se observa no cenário nacional 
que, apesar da existência de medidas consistentes para mensurar a personalidade, como, por exemplo, o Inventário de Cinco Fatores Revisado - NEO-PI-R (Costa \& McCrae, 2007) e a Bateria Fatorial de Personalidade - BFP (Nunes, Hutz \& Nunes, 2010), aceitas no Sistema de Avaliação de Testes Psicológicos (SATEPSI), até o momento, evidencia-se a carência de medidas com amostras de normatização, nas quais figurem profissionais da segurança pública.

Partindo da compreensão de que testes psicológicos são capazes de oferecer informações sobre o funcionamento dos processos mentais (Urbina, 2007) e da constatação de que sua utilidade encontra-se vinculada à apresentação de qualidades psicométricas e evidências de validade (Primi, Muniz \& Nunes, 2009), a presente pesquisa objetivou a construção de uma escala para avaliar instabilidade emocional no contexto da segurança pública. Buscaram-se também evidências de validade por meio da análise de conteúdo dos itens, evidências com base na estrutura interna dos mesmos e na relação com variáveis externas, nesse caso, convergência com uma escala que avalia o mesmo construto, além da verificação da confiabilidade da escala por meio da análise da consistência interna (alfa de Cronbach).

\section{Método}

Para construção e validação da Escala de Instabilidade Emocional para a Segurança Pública - IESP foi adotado o modelo proposto por Pasquali (2010), por meio de dois estudos. O primeiro estudo realizado tratou da construção dos itens da IESP e de sua análise teórica e semântica (evidência de conteúdo). O segundo destinou-se a demonstrar os índices de precisão da IESP, por meio do cálculo do alfa de Cronbach, assim como explicitar as evidências de validade da IESP com base na estrutura interna e relacionada com variáveis externas.

\section{$1^{\circ}$ Estudo: Construção da Escala de Instabilidade Emocional para a Segurança Pública IESP}

Inicialmente, o projeto de construção da IESP foi submetido e aprovado pelo Comitê de Ética em Pesquisa da Universidade Salgado de Oliveira; em seguida, foi solicitada a autorização para realização da pesquisa com o efetivo de uma instituição de segurança pública do estado do Rio de Janeiro. Para a composição dos itens do instrumento foram utilizadas três fontes: a) os termos oriundos da revisão da literatura da área; b) as expressões, frases e exemplos produzidos em um grupo focal e c) o levantamento realizado junto aos itens de testes de personalidade de medida correlata.

$\mathrm{Na}$ pesquisa realizada, instabilidade emocional é identificada como um traço que caracteriza pessoas que apresentam maior propensão para vivenciar emoções negativas, apresentar baixo autocontrole e para perceber o mundo como perigoso e ameaçador (Costa \& McCrae,1995; Eysenck, 1990; McCrae, 2006; Sangil \& Weed, 2007; Smith, Bond \& Kâğıtçıbaşı, 2006). Em tais estudos foram apontados seis indicadores que expressam as facetas teóricas do fator de instabilidade emocional: ansiedade; raiva e/ou hostilidade; depressão; embaraço e/ou constrangimento; impulsividade; vulnerabilidade (Costa \& McCrae, 2007). Tais indicadores, ainda que com diferentes nomenclaturas, também foram encontrados nos testes de personalidade ICFP-R (Pasquali, Vasconcelos \& Tróccoli, 2004); NEO PI-R (Costa \& McCrae, 2007) e Bateria Fatorial de Personalidade - BFP (Nunes, Hutz \& Nunes, 2010).

Por outro lado, buscando delinear o construto instabilidade emocional para o contexto da segurança pública, foi realizado um grupo focal, com oito servidores da área, no qual foram levantados exemplos de como os mesmos identificavam situações consideradas como indicadores de instabilidade emocional. Nos exemplos fornecidos, foram apontadas situações onde instabilidade emocional pôde ser caracterizada como a apresentação de hostilidade para com os colegas e com a população; descontrole quando confrontado com situações de forte tensão emocional e aumento da irritabilidade quando diante de situações corriqueiras e uso abusivo de álcool e outras substâncias. O grupo em questão também contribuiu para a compreensão de que tais características são consideradas, no meio profissional, como indicativos de maior dificuldade no cumprimento de missões, influenciando diretamente na qualidade do trabalho e na vida pessoal de profissionais de segurança pública.

A partir da análise dos dados apresentados, foram elaboradas 133 frases, nas quais foram aproveitados exemplos fornecidos no grupo focal. Esses itens foram então submetidos à avaliação de sete participantes de um grupo de pesquisa, composto por alunos de mestrado da área de avaliação psicológica. Para realização de tal análise, foi solicitado a cada avaliador que aferisse quatro quesitos: a) se os itens apresentados guardavam relação com o fator de instabilidade emocional; b) que indicassem a que faceta se relacionava o item; c) que definissem se os itens 
eram compreensíveis e d) que, quando julgado necessário, apresentassem sugestões para o aprimoramento das frases. O grupo sugeriu modificações em alguns itens e a criação de mais frases para que os indicadores fossem suficientemente explorados. Foram criados então mais 16 itens, passando o instrumento a contar com 149 componentes, que foram submetidos a uma segunda avaliação, objetivando a delimitação do instrumento final.

$\mathrm{Na}$ segunda avaliação participaram dez juízes, todos profissionais com conhecimento teórico e prático na medida da personalidade. Cada juiz recebeu um instrumento contendo os itens para análise, bem como as definições constitutivas dos indicadores que seriam avaliados. Os 149 itens foram divididos em três versões (A, B e C), sendo duas com 50 e uma com 49 itens aleatoriamente divididos. A subdivisão dos itens em três versões objetivou diminuir o desgaste com a realização da tarefa e com isso evitar possíveis prejuízos à análise. A versão " $A$ " foi respondida por quatro juízes e as versões "B" e "C" foram respondidas por três juízes cada uma. O critério utilizado para manutenção dos itens, na versão final do instrumento, foi a apresentação de, pelo menos, $80 \%$ de concordância entre os avaliadores. Ao final dessa avaliação, foi observada a necessidade de suprimir nove itens e modificar outros 14 itens para que se tornassem mais claros. Dessa forma, o instrumento final foi constituído por 140 componentes.

Posteriormente, foram construídas instruções sobre como responder à escala e o instrumento foi então submetido à análise semântica, para verificação de sua adequação à população-alvo. Tal análise consistiu na aplicação do mesmo a um grupo formado por 18 profissionais de diferentes cargos de uma instituição da área da segurança pública da cidade do Rio de Janeiro. Aos participantes da análise semântica foi solicitado que avaliassem a clareza dos itens, bem como das instruções apresentadas. Para isso foi utilizada a escala tipo likert de 5 pontos ( $1=$ pouquíssimo; $2=$ pouco; $3=$ médio; $4=$ muito e $5=$ muitíssimo).

\section{Resultados}

Os dados indicaram que $83 \%$ dos participantes julgaram os itens como adequados $(61 \%=$ muitíssimo claros; $22 \%$, claros) e $17 \%$ dos participantes consideraram os itens como pouco claros. Quanto às instruções, $50 \%$ dos participantes as julgaram muitíssimo claras; $33 \%$ as consideraram muito claras; $11 \%$, como medianas, e $6 \%$ consideraram as instruções como pouquíssimo claras. Por fim, o grupo considerou que a modificação de 2 frases contribuiria para o aumento da clareza das mesmas e tal sugestão foi acatada.

$2^{\circ}$ Estudo: Consistência e evidências de validade da IESP com base na estrutura interna e na relação com variáveis externas

O segundo estudo de validade da IESP foi realizado por meio de duas etapas distintas. A primeira objetivou apresentar evidências de validade baseadas na estrutura interna da escala, bem como dados de sua precisão. A segunda etapa visou à apresentação de evidências de validade da IESP por meio da relação com outras medidas.

\section{$1^{a}$ Etapa-Evidências de validade baseadas na estru- tura interna da escala}

\section{Participantes}

Participaram desta etapa do estudo 1.601 profissionais de uma instituição da área de segurança pública do estado do Rio de Janeiro, sendo que, desses, 1.278 eram homens (79,8\%), 304, mulheres (19\%) e 1,2\% não respondeu. A diferença percentual entre homens e mulheres reflete a mesma relação verificada no efetivo de profissionais da área. As idades variaram entre $18 \mathrm{e}$ 55 anos $(\mathrm{M}=27,1 ; \mathrm{DP}=4,73)$. Quanto à escolaridade dos participantes, 799 sujeitos apresentaram o ensino médio completo (49,9\%), 459 participantes apresentaram o nível superior incompleto (28,7\%), 275 sujeitos apresentaram o nível superior completo $(17,2 \%)$ e 40 alcançaram a pós-graduação $(2,5 \%)$.

\section{Instrumento}

\section{- Escala de Instabilidade Emocional para a Segurança Pública (IESP)}

Para realização do estudo de validação, foi utilizada a escala denominada de Escala de Instabilidade Emocional para a Segurança Pública - IESP. Tal instrumento foi composto por um caderno de aplicação com 140 itens, acrescido de um formulário de coleta de dados socioculturais e de trabalho e de um cartão de respostas para leitura ótica. A página de rosto da IESP continha as instruções necessárias para seu correto preenchimento, por meio de uma escala likert de 7 pontos $(7=$ representa-me totalmente/Descreve totalmente a 1=Não me representa de forma alguma/ Não tem nada a ver comigo). 


\section{Procedimento}

Todos os participantes foram informados dos objetivos do estudo e assinaram termo de consentimento livre esclarecido. A aplicação do instrumento foi realizada de forma coletiva e os participantes receberam instruções padronizadas (orais e por escrito) sobre como responder ao instrumento, sendo também cientificados sobre o sigilo das informações. Em média, o tempo gasto para resposta à IESP foi de 40 minutos, incluída a marcação da folha de leitura óptica.

\section{Resultados}

Inicialmente foi realizada uma análise dos componentes principais (PC), para verificar a fatorabilidade da Matriz, sendo verificado que o índice de fatorabilidade mostrou um KMO de 0,97. Foi, então, realizada a extração de 11 fatores pelo método Principal Axis Factoring (PAF), com rotação oblíqua direct oblimim, tratamento de casos omissos pelo método pairwise e supressão de cargas fatoriais inferiores a 0,30 (Hair, Black, Babin, Anderson \& Tatham, 2009). Nessa análise optou-se pela exclusão de 24 itens que apresentaram carga menor que 0.30. Em uma segunda PAF, na qual foram extraídos entre quatro e onze fatores, verificou-se que a estrutura mais parcimoniosa e que concentrou maior consistência teórica foi a que contemplou quatro fatores.

Para a presente análise, em entendimento com a literatura da área, os fatores são identificados como facetas que compõem o fator de instabilidade emocional. Nesse sentido, a análise da estrutura da IESP, julgada como mais adequada, aponta que suas quatro facetas explicam, juntas, $36 \%$ da variância, com apenas $7 \%$ da dispersão da matriz de correlação continuando não explicada. $\mathrm{Na}$ Tabela 1 , verifica-se que as quatro facetas apresentam correlações superiores a 0,30, o que indica a existência de um fator de segunda ordem comum a todas (Pasquali, 2008).

$\mathrm{Na}$ análise da estrutura com quatro facetas, dezessete itens apresentaram cargas menores que 0,30, e, por isso, também foram excluídos da escala, de forma que a matriz fatorial da IESP apresenta 99 itens que compõem o seu fator geral, ostentando cargas fatoriais entre 0,31 e 0,75 . A primeira faceta é composta por 36 itens com cargas fatoriais entre 0,31 e 0,73 e que explicam $33,10 \%$ da variância. A segunda faceta é composta por 23 itens e explica 3,95\% da variância com cargas fatoriais entre 0,31 e 0,73. 26 itens com cargas fatoriais compreendidas entre 0,30 e $0,72 \mathrm{com}-$ põem a terceira faceta, que explica $3,16 \%$ do modelo. A última faceta é composta por 14 itens com cargas variando entre $-0,31$ e $-0,58$ e que explica $2,72 \%$ da variância. Os autovalores, percentual de variância e

Tabela 1. Correlação entre as quatro facetas

\begin{tabular}{lcccc}
\hline Fator & 1 & 2 & 3 & 4 \\
\hline 1 & 1,00 & & & \\
2 & 0,47 & 1,00 & 1,00 & \\
3 & $-0,54$ & $-0,41$ & 0,34 & 1,00 \\
4 & $-0,37$ & $-0,40$ & & \\
\hline
\end{tabular}

Nota: Método de Extração: PAF, com rotação oblimin.

Tabela 2. Autovalor, percentual de variância e número de itens - extraído da matriz da IESP $(\mathrm{N}=1601)$

\begin{tabular}{lccccc}
\hline Itens & Fator Geral & \multicolumn{5}{c}{ Facetas } \\
\hline & $\mathrm{I}$ & 1 & 2 & 3 & 4 \\
Autovalor & 28,28 & 32,77 & 3,91 & 3,13 & 2,69 \\
$\%$ da Variância & 28,56 & 33,10 & 3,95 & 3,16 & 2,72 \\
$\mathrm{~N}^{\text {o de itens }}$ & 99 & 36 & 23 & 26 & 14 \\
\hline
\end{tabular}


número de itens - extraído da matriz da IESP, estão descritos na Tabela 2.

Para análise da consistência dos componentes do instrumento foi calculado o alfa de Cronbach $(\alpha)$. Os resultados demonstram que os coeficientes apresentaram variação de 0,88 a 0,93 , sendo que o índice de precisão do fator geral foi de 0,91 , seguido pelos índices de 0,93 para a faceta $1 ; 0,88$ na faceta $2 ; 0,91$ na faceta 3 e 0,91 na faceta 4 . Tais dados indicam que a IESP apresenta bons índices de precisão e apontam para a confiabilidade de seus resultados (Pasquali, 2003).

Para a análise dos componentes da IESP, foi examinado o conteúdo de seus itens e realizada a interpretação de seu fator geral, bem como de cada uma de suas quatro facetas. $\mathrm{O}$ fator geral (instabilidade emocional) descreve sujeitos que, em geral, tendem a apresentar características compatíveis com a acentuação de depressão, ansiedade, irritabilidade e baixa estabilidade emocional. A faceta F1 (depressão) é composta por 36 itens e se refere a indivíduos que tendem a apresentar sentimentos de desesperança, tristeza, baixa autoestima, desânimo e dificuldade em lidar com situações de autoexposição. A faceta F2 (ansiedade) é composta por 23 itens e descreve sujeitos que, em suas experiências e contatos interpessoais, demonstram preocupação exacerbada com eventos não usuais, muitas vezes experimentando sofrimento com a antecipação dos mesmos. A faceta F3 (controle emocional) é composta por 26 itens e descreve sujeitos caracterizados como emocionalmente estáveis, que vivenciam adequadamente as diversas situações da vida e apresentam facilidade em manter o autocontrole e maior resistência ao estresse. A Faceta f4 (irritabilidade) é composta por 14 itens e descreve sujeitos que muito facilmente se aborrecem, tendendo a perder o controle das emoções e ofender física e moralmente às demais pessoas. A tendência a agir sem prévia reflexão, não raro, permite que sujeitos, assim caracterizados, envolvam-se em problemas.

\section{$2^{a}$ Etapa - Evidências de validade baseadas na relação com va- riáveis externas - validade convergente}

$\mathrm{Na}$ definição contemporânea de validade, um dos aspectos considerados para análise de evidências de validade se refere à evidência baseada em variáveis externas, nas quais se verifica a validade convergente entre a medida avaliada e outras variáveis, que teoricamente devem estar relacionadas (Primi, Muniz \& Nunes, 2009). Para análise da evidência de validade da IESP, foi investigada a correlação dessa medida com a Escala Fatorial de Ajustamento Emocional/Neuroticismo - EFN.

\section{Participantes}

Participaram deste estudo 322 profissionais de uma instituição da área de segurança pública do estado do Rio de Janeiro, sendo que destes, 272 sujeitos eram homens $(84,5 \%)$ e 44 eram mulheres $(13,7 \%)$ com idades variando entre 19 e 35 anos $(M=26,7 ; D P=3,27)$. Quanto à escolaridade, 180 participantes tinham ensino médio completo (56\%), 84 participantes apresentaram nível superior incompleto (26\%), 46 sujeitos concluíram o nível superior completo $(14,3 \%)$ e 2 apresentaram pós-graduação $(0,6 \%)$.

\section{Instrumentos}

Para essa análise foram utilizados os 99 itens da IESP que resultaram da análise fatorial e a Escala Fatorial de Ajustamento Emocional/Neuroticismo EFN (Hutz \& Nunes, 2001). A EFN foi validada com uma amostra de 1.176 estudantes universitários de vários estados brasileiros. Ela se baseia no modelo dos Cinco Grandes Fatores e é constituída por 82 itens respondidos por meio de uma escala tipo likert de 7 pontos $(1=$ completamente inadequada a $7=$ perfeitamente adequada). Sua avaliação é feita com base em uma estrutura composta por quatro facetas e um fator geral. O fator geral da EFN (neuroticismo) apresenta um alfa de 0,94 e se refere à expressão de características compatíveis com presença de vulnerabilidade, desajustamento psicossocial, ansiedade e depressão. A subescala de vulnerabilidade apresenta um alfa de 0,89 e é composta por 23 itens que expressam medo, insegurança, indecisão, entre outros. A faceta de desajustamento psicossocial apresenta alfa de 0,82 e compreende 14 itens, cujo conteúdo refere-se, entre outras coisas, à tipificação de comportamentos considerados socialmente inadequados. A faceta de ansiedade apresenta alfa de 0,87 , sendo composta por 25 itens que descrevem sintomas associados com o transtorno de ansiedade. A faceta de depressão apresenta alfa de 0,87 e é composta por 20 itens que se referem a sintomas relacionados com desesperança e fantasias associadas ao suicídio.

\section{Procedimentos}

Para a efetivação da presente etapa, foi realizada a aplicação concomitante de ambos os instrumentos, sendo inicialmente respondida a IESP e, em seguida, a EFN, conforme as normas previstas no manual de 
aplicação da mesma. Consequentemente, os procedimentos já descritos na etapa de validação da estrutura interna da IESP foram igualmente observados.

\section{Resultados}

Para o presente estudo, foram calculados os escores de cada uma das facetas do instrumento EFN, bem como de seu fator geral. Posteriormente, foi calculado o coeficiente de correlação de Pearson, para verificação das correlações encontradas entre a IESP e a EFN, conforme apresentado na Tabela 3.

$\mathrm{Na}$ matriz de correlações entre as duas medidas, é possível observar a forte correlação positiva $(0,70)$ entre o fator geral da IESP - instabilidade emocional e o fator geral da EFN - neuroticismo. Tal dado indica que sujeitos que exibem traço elevado de instabilidade emocional de modo análogo o apresentarão em neuroticismo.

Também foi verificado que as facetas da EFN, vulnerabilidade e ansiedade, apresentaram correlações positivas superiores a $0,60 \mathrm{com}$ as facetas da IESP de depressão (F1), ansiedade (F2) e irritabilidade (F4). Quanto à faceta de desajustamento psicossocial da EFN, esta apresentou correlações positivas e significativas (superiores a 0,32 ) com as referidas facetas da IESP. Quanto à faceta de controle emocional da IESP, notou-se uma correlação negativa com os componentes da EFN (vulnerabilidade $=-0,46$; ansiedade $=-0,46$ e desajustamento psicossocial=-0,34). No tocante à faceta de depressão da EFN, foi observado que a mesma apresentou correlações fracas com todos os componentes da IESP (depressão - F1=0,23; Ansiedade $-\mathrm{F} 2=0,20$; irritabilidade $-\mathrm{F} 4=0,20$ ), à exceção da faceta de controle emocional, com a qual depressão não se correlacionou significativamente.

\section{Discussão}

$\mathrm{Na}$ análise dos componentes da IESP, verificou-se, como solução mais parcimoniosa, estrutura composta por um fator geral e quatro facetas. De modo análogo, estudo realizado no Brasil verificou que o fator neuroticismo, quando pesquisado em uma amostra brasileira, apresentou como melhor solução teórica a definição de um fator geral e quatro facetas (Hutz \& Nunes, 2001).

Embora esses resultados difiram daqueles encontrados no NEO-PI-R, medida que na atualidade é a mais amplamente utilizada na avaliação dos Cinco Grandes Fatores da personalidade e onde o fator de neuroticismo apresenta seis facetas, não há na literatura indicação taxativa quanto à quantidade ideal dessas facetas (Hutz \& Nunes, 2001). Não obstante, no modelo apresentado no presente trabalho, mantém-se a compreensão de que a personalidade pode ser mais bem compreendida por meio da análise de facetas, e não apenas dos fatores (Costa \& McCrae, 1995).

A análise dos estudos apresentados torna possível deduzir a consistência teórica da IESP, na medida em que as quatro facetas, conforme demonstrado, referem-se ao mesmo construto subjacente e apresentam características associadas ao fator de instabilidade emocional. A fidedignidade da IESP foi afirmada por meio da apresentação dos coeficientes de consistência interna, considerados robustos e que atestam a precisão da escala (Pasquali, 2003).

$\mathrm{Na}$ verificação de validade convergente, observou-se que as correlações entre as facetas da IESP e as da EFN apresentaram-se entre altas e moderadas. A correlação observada entre o fator geral da IESP e da EFN indica que sujeitos que apresentam elevado traço de instabilidade emocional também apresentarão em neuroticismo. Tal resultado é relevante, pois contempla

Tabela 3. Correlações entre IESP e EFN

\begin{tabular}{lccccc}
\hline & \multicolumn{5}{c}{ EFN } \\
\cline { 2 - 5 } IESP & Neuroticismo & Vulnerabilidade & Ansiedade & Depressão & $\begin{array}{c}\text { Desajustamento } \\
\text { Psicossocial }\end{array}$ \\
\hline Depressão (F1) & $0,67^{* *}$ & $0,58^{* *}$ & $0,66^{* *}$ & $0,23^{* *}$ & $0,49^{* *}$ \\
Ansiedade (F2) & $0,67^{* *}$ & $0,65^{* *}$ & $0,66^{* *}$ & $0,20^{* *}$ & $0,32^{* *}$ \\
Controle (F3) & $-0,48^{* *}$ & $-0,46^{* *}$ & $-0,46^{* *}$ & $-0,05$ & $-0,34^{* *}$ \\
Irritabilidade (F4) & $0,62^{* *}$ & $0,53^{* *}$ & $0,63^{* *}$ & $0,20^{* *}$ & $0,42^{* *}$ \\
Instabilidade emocional (FG) & $0,70^{* *}$ & $0,64^{* *}$ & $0,69^{* *}$ & $0,20^{* *}$ & $0,45^{* *}$ \\
\hline
\end{tabular}

**Correlação significante $\operatorname{com} p<0,01$ 
o pressuposto de que ambas as medidas mensuram o mesmo construto e apresenta um indício de validade convergente entre os dois instrumentos (Anastasi \& Urbina, 2000; Pasquali, 2010).

As correlações observadas entre as facetas de vulnerabilidade, ansiedade e desajustamento psicossocial da EFN, além das facetas da IESP de depressão, ansiedade e irritabilidade foram positivas e significativas. Tal dado possibilita afirmar que quanto mais triste e desanimado (depressão F1), tenso apreensivo e medroso (ansiedade F2) e facilmente irritável (irritabilidade F3) for o sujeito, mais vulnerável, ansioso e desajustado psicossocialmente ele também se apresentará. Tais dados apontam que os elementos constituintes de ambas as escalas, encontram-se estreitamente relacionados. Esses dados corroboram os achados de Hutz e Nunes (2001) e Vasconcelos (2005), que identificaram, em pesquisas nacionais, a utilidade do modelo dos cinco grandes fatores CGF para o estudo da personalidade, bem como a robustez da proposta de estrutura da instabilidade emocional.

Já entre a faceta de controle emocional da IESP e as facetas de vulnerabilidade, ansiedade e desajustamento psicossocial da EFN, as correlações foram negativas e significativas. Isso também contempla a expectativa de que pessoas que apresentam controle sobre suas emoções se mostrem menos vulneráveis, ansiosas e desajustadas psicossocialmente.

A faceta de depressão da EFN apresentou correlações fracas com todos os componentes da IESP, sendo que no tocante à faceta de controle emocional, a correlação verificada não foi significativa. Isso indica que os comportamentos que caracterizam a faceta de depressão da EFN guardam pouca relação com as facetas e fator da IESP. Tais resultados são inesperados, principalmente pelo padrão de correlações observado entre os demais componentes de ambos os instrumentos.

A análise das correlações entre as facetas de depressão dos dois instrumentos, ainda que concorde com a informação de que a faceta de depressão da EFN pouco se relaciona à IESP, destaca-se por confrontar a expectativa de que ambas mensurassem os mesmos aspectos. Uma possível explicação para tal fenômeno pode ser buscada por meio da análise da composição dos itens de ambas as facetas. Foi observado que a faceta de depressão da EFN caracteriza aspectos compatíveis com fantasias de suicídio, como apresentado nos exemplos dos itens 35 (já tentei cometer suicídio) e 39 (já falei para outras pessoas que iria cometer suicídio). Tais elementos, bem como o componente explícito de interrupção da própria vida, não foram identificados nos itens que compõem a faceta de depressão da IESP, cuja composição aponta para aspectos relacionados à baixa autoestima (minha autoestima é baixa); tristeza (minha vida se mostra cheia de tristeza) e desânimo (sinto-me profundamente desanimado).

Outra análise possível refere-se à constatação de que itens que incorporam "sintomas de desesperança, depressão, ideação suicida e similares" (Hutz \& Nunes, 2001, p. 12) talvez apresentem baixa aderência, quando apresentados em situações tais como a do presente estudo, realizado com amostra específica de profissionais. Vasconcelos (2005) também identificou que os itens que compõem a faceta de depressão da EFN podem se mostrar úteis para avaliações clínicas, mas que os mesmos não se mostram adequados ao contexto de seleção de pessoal ou ao uso no ambiente de trabalho, uma vez que facilitam a manipulação das respostas, por serem consideradas mal vistas pelos candidatos.

Finalmente, destaca-se que um dos aspectos contemplados na busca por evidências de validade refere-se ao incremento de informações sobre a aplicabilidade de instrumentos em diferentes contextos (Nunes \& Primi, 2010). Nesse sentido, os dados apresentados podem ser considerados como um acréscimo de evidência de validade para a EFN no contexto da segurança pública.

\section{Considerações finais}

Tendo por base todos os indícios apresentados, pode-se concluir que a IESP se mostrou como um instrumento válido. Essa escala tem por finalidade contribuir com a ciência psicológica ao apresentar uma nova proposta de instrumento de medida da personalidade, bem como contribuir para o campo da medida, por meio da disponibilização de um teste com validade para uma população cuja relevância, apesar de já demonstrada, não era contemplada em estudos específicos.

Uma vez que o objetivo central da IESP é servir como uma medida da instabilidade emocional no contexto da segurança pública, espera-se que a mesma seja utilizada na realização de avaliações psicológicas para ingresso na área. Ademais, espera-se que o instrumento em questão possa oferecer estudos futuros sobre essa população na medida do construto de instabilidade emocional.

Quanto à baixa correlação encontrada entre a faceta de depressão da EFN e as facetas e fator geral da IESP, identifica-se a necessidade de estudos adicionais que possam melhor avaliar esses dados. Propõe-se, 
ainda para o novo instrumento proposto, análises confirmatórias e estudos baseados na Teoria de Resposta ao Item (TRI), para incremento de informações sobre a discriminação dos itens da IESP.

Tais pesquisas também atenderão a outras necessidades levantadas nos estudos realizados para validação da IESP. A primeira refere-se à ampliação da abrangência da medida com a inclusão de amostras de profissionais de diversos estados brasileiros e de variados segmentos da segurança pública. Outro desafio se refere à construção de versões reduzidas da IESP, a fim de minimizar o desgaste com a resposta a grande quantidade de itens, o que poderia inviabilizar sua aplicação concomitante com outras medidas em avaliações psicológicas de processos seletivos.

\section{Referências}

Anastasi, A. \& Urbina, S. (2000). Testagem psicológica. Porto Alegre: Artmed.

Bishop, G. D., Tong, E. M. W., Diong, S. M., Enkelmann, H. C., Why, Y. P., Khader, M., \& Ang, J. C. H. (2001). The relationship between coping and personality among police officers in Singapore. Journal of Research in Personality, 35, 353-374.

Brasil (1988). Constituição da República Federativa do Brasil. Brasília, Distrito Federal.

Cochrane R. E., Tell, R. P., \& Vandecreek, L. (2003). Psychological testing and the selection of police officers: a national survey. Criminal Justice and Behavior 30(5), 511-537.

Costa, P. T., \& McCrae, R. R. (1995). Domains and facets: hierarchical personality assessment using the revised neo personality inventory. Journal of Personality Assessment, 64(1), 21-50.

Costa, P. T., \& McCrae, R. R. (2007). NEO PI-R - inventário de personalidade neo revisado e inventário de cinco fatores NEO revisado - NEO-FFI-R (Versão Curta). São Paulo: Vetor.

Eysenck, H. J. (1990). Biological dimension of personality. Em L. A. Pervin (Ed.), Handbook of personality - theory and research (pp. 244-276). Nova Iorque: The Guilford Press.

Faiad, C. (2007). Como viabilizar nossos testes em um mercado como esse? Em Anais do III Congresso Brasileiro de Avaliação Psicológica (p.112) Brasil: João Pessoa.
Hair, J. F., Black, W. C., Babin, B. J., Anderson, R. L., \& Tatham, R. L. (2009). Análise multivariada de dados. $6^{a}$ Edição. (A. S. Sant'Anna, Trad.). São Paulo: Bookman.

Hampson, S. E. (2012). Personality process: mechanisms by which personality traits. Annual Review of Psychology, 63, 315-319.

Ho, T. (2001). The interrelationships of psychological testing, psychologists recommendations, and police departments recruitment decisions. Police Quarterly, 4, 318-342.

Hutz, C. S., \& Nunes, C. H. S. (2001). Escala fatorial de ajustamento emocional/neuroticismo EFN. São Paulo: Casa do Psicólogo.

McCrae R. R. (2006). O que é personalidade? Em C. Flores-Mendonza \& R. Colom (Org.). Introdução à psicologia das diferenças individuais (pp. 203-218). Porto Alegre: Artmed.

McCrae R. R., \& Costa, P.T. (1989). Rotation to maximize the construct validity of factors in the neo personality inventory. Multivariate Behavioral Research, 2(1), 107-124.

Moura, C. F. (2008). Reação à frustração: construcão e validação da medida e proposta de um perfil de reação. Tese de Doutoramento. Universidade de Brasília.

Noronha, A. P. P., \& Alchieri, J. C. (2005). Reflexões sobre instrumentos de avaliação psicológica. Em Primi, R. (Org.), Temas em avaliação psicológica. (pp. 19-36). São Paulo: Casa do Psicólogo.

Nunes, C. H. S., \& Primi, R. (2010). O SATEPSI: desafios e projetos de aprimoramento. Em Conselho Federal de Psicologia (Org.), Avaliação psicológica: diretrizes na regulamentaşão da profissão. (pp. 129-148). Brasília - DF: CFP.

Nunes, C. H. S., Hutz, C. S. \& Nunes, M. F. O. (2010). Bateria fatorial de personalidade. São Paulo: Casa do Psicólogo.

Pasquali, L. (2003). Psicometria: a teoria dos testes na psicologia e na educação. Petrópolis: Vozes.

Pasquali, L. (2008). Análise fatorial para pesquisadores. Brasillia: LabPAM-UNB.

Pasquali, L. (2010). Testes referentes a construto: teoria e modelo de construção. Em L. Pasquali (Org.). instrumentação psicológica: fundamentos e práticas (pp. 165-198). Porto Alegre: Artmed. 
Pasquali, L., Vasconcelos, T. S., \& Tróccoli, B. T. (2004). ICFP-R - Inventário reduzido dos cinco fatores de personalidade: manual técnico e de aplicação. Distrito Federal: LabPAM- Universidade de Brasília.

Pasquali, L., Cabral, A. R., Figueira, K. S., Rodrigues, M. M. M., \& Moura, C. F. (2002). Profissiografia do cargo de agente de policia civil do Distrito Federal. SENASP. Disponível em: www.mj.gov.br/senasp/monografias. Acessado em: 22 jan. 2006.

Primi, R., Muniz, M., \& Nunes, C. H. S. S. (2009). Definições contemporâneas de validade de testes psicológicos. Em C. S. Hutz (Org.). Avanços e polêmicas em avaliação psicológica (pp. 243-265). São Paulo: Casa do Psicólogo.

Sangil, K., \& Weed, C. (2007). Neuroticism. Encyclopedia of socialpsychology. Disponível em: http://sageereference.com/socialpsychology/Article_n370.html. Acessado em: 27 abril. 2010.

Smith, P. B., Bond, M. H., \& Kâğıtçıbaşı, C. (2006). Understanding social psychology across cultures: living and working in a changing world. Londres: SAGE.

Thadeu, S. H (2011). A validade da avaliação psicológica em um processo seletivo na área de segurança pública. Dissertação de Mestrado. Universidade Salgado de Oliveira.
Thadeu, S. H., Kistenmacker, E. R. O., \& Reis, M. S. (2009). Quer passar fácil?: banalização dos testes psicológicos e o prejuízo em seleções de policiais. Em Anais do IV Congresso Brasileiro de Avaliação Psicológica. Brasil: Universidade São Francisco.

Thadeu, S. H., Reis, M. S., Moura, C. F., Caetano, P. F., Braga, J. L., \& Pasquali, L. (2008). Profissiografia dos soldados policiais militares da policia militar do Estado do Rio de Janeiro - Brasil. XIII Conferência Internacional de Avaliação Psicológica: Formas e Contextos (p. 46). Portugal: Uminho.

Urbina, S. (2007). Fundamentos da testagem psicológica. Porto Alegre: Artmed

Vasconcelos, T. S. (2005). O Inventário fatorial dos cinco fatores de personalidade no ambiente de trabalho. Tese de Doutoramento. Universidade de Brasília.

Wiggins, S. J., \& Pincus, A. L. (1992). Personality: structure and assessment. Annual Reviews of Psychology.43, 473-504.

Recebido em: 29/01/2013

Reformulado em: 18/09/2013 Segunda reformulação em: 16/10/2013

Aprovado em: 12/11/2013

Sobre as autoras:

Marcela dos Santos Reis é mestre em Psicologia pela Universidade Salgado de Oliveira, pós-graduada em Desenvolvimento de Recursos Humanos em Saúde pela Escola Nacional de Saúde Pública e graduada em Psicologia pela Universidade do Estado do Rio de Janeiro. Possui experiência na Coordenação de Avaliação Psicológica em concursos públicos e na docência na área de elaboração de medidas psicológicas. Curriculum Lattes: http://lattes.cnpq. br/8919486454664329

E-mail: mdsreis@ig.com.br

Cristiane Faiad é doutora e mestre em Psicologia pela Universidade de Brasília. Atualmente é professora titular do Programa de Mestrado em Psicologia da Universidade Salgado de Oliveira, coordenadora do Grupo de Pesquisa em Avaliação Psicológica em Segurança Pública e trabalha com construção e validação de instrumentos, análise profissiográfica, mapeamento de competências e na área de gestão de pessoas. Curriculum Lattes: http://lattes.cnpq. br/5240057556076011

E-mail: crisfaiad@gmail.com

Contato com as autoras:

Rua Dr. Julio Otoni, 351 Ap. 101 - Santa Teresa - Rio de Janeiro - RJ CEP: 20.241-400 